\title{
Synthesis and Characterization of Photoluminescent PVA/ZnS: Mn2+ Nanocomposites
}

Priya $\mathrm{L}^{*}$

\section{Abstract}

A $\mathrm{ZnS}$ nanoparticle doped with $\mathrm{Mn} 2+$ is synthesized in aqueous media and PVA using chemical co-precipitation method. This colloid was analyzed using uv-vis spectrophotometry. It is observed that the absorption peak blue shifts as compared to the bulk absorption of ZnS suggesting the nanoparticle formation. The energy gaps of these nanoparticles were calculated from the uvvis spectra. The average particle size analysis is carried out using XRD. Photoluminescence of PVA/ ZnS: $\mathrm{Mn}^{2+}$ is studied. It is observed that the composite sample exhibits an orange emission peak as is reported for pure $\mathrm{ZnS}$ : $\mathrm{Mn} 2^{+}$.

Keywords: Nanoparticles, ZnS: $\mathrm{Mn}^{2+}, \mathrm{XRD}$, Photoluminiscence

\section{Introduction}

Luminescent nanoparticles with advantageous properties for optoelectronics applications are available in both inorganic and organic forms. In recent years a wide range of materials including metals, semiconductors and metal oxides are synthesized in nanometer scale. In particular II-VI semiconductor nanocrystals (NCs) are receiving a lot of attention due to their potential application in optoelectronics and photonics. The colour tunability

\footnotetext{
* Department of Physics, Centre for Postgraduate Studies, Jain University, III block Jayanagar, Bangalore-560 011; svl_priya@yahoo.com
} 
of these semiconductor nanoparticles as a function of size is one of their most attractive properties. But a lot of issues needs to be addressed before it can be brought to end use. In that sense, the current bottle-neck of these materials is their poor compatibility with micropatterning methods in general and MEMS processes in particular. Inorganic nanoparticles dispersed in polymer matrix may lead to versatile nanoscale devices. Colloidal methods provide effective routes to prepare semiconductor nanoparticles that are dispersible in organic solvents. Recently the focus is towards the fabrication of polymer nanocomposites containing micrometer or nanometer sized inorganic semiconductor. This is due to the good processability and the structural properties of the polymeric matrix, and the unique optoelectronic characteristics of the inorganic moiety which can be used for many purposes such as optical switches, sensors, electro-luminescent devices, lasers and biomedical tags. Inorganic semiconductor nanocrystals (NCs), primarily based on CdSe/ZnS, are highly efficient light-emitters in the visible range of the spectrum. These nanoparticles when integrated with polymers can lead to a lot of applications [1-4].

The incorporation of inorganic particles into polymers allows one to integrate new functions inside polymer matrices. Modification of the matrix by dispersing a second inorganic component into the polymer typically results in a significant loss of transparency due to scattering from large particles or agglomerates. Incorporation of smaller particle sizes can restore the transparency of the polymer selected as well as add new functionalities to the polymer composite. The general method adopted is to prepare the composites using in-situ polymerization techniques in a solution containing the particle dispersion. Here we have chosen a simple as prepared partially crystalline PVA which is a water soluble polymer as the matrix without reverting to the traditional insitu synthesis technique.

\section{Experimental}

Nanoparticles of $\mathrm{Mn}^{2+}$-doped $\mathrm{ZnS}$ were prepared by chemical coprecipitation method. All the chemicals were of AR grade. Freshly prepared aqueous solutions of the chemicals were used for the synthesis of nanoparticles. These particles were prepared at room 
temperature by dropping simultaneously $20 \mathrm{ml}$ of $0.4 \mathrm{M}$ solution of zinc sulphate, $20 \mathrm{ml}$ of $0.1 \mathrm{M}$ solution of manganese sulphate and $20 \mathrm{ml}$ of $0.5 \mathrm{M}$ solution of sodium sulphide into $40 \mathrm{ml}$ of distilled water containing $20 \mathrm{ml}$ of $0.1 \mathrm{M}$ solution of EDTA which was vigorously stirred using a magnetic stirrer. The role of EDTA was to stabilize the particles against aggregation of particles. The precipitate was separated from the reaction mixture, washed several times with distilled water. The wet precipitate was dried and thoroughly ground [5]. The same procedure is used to synthesize ZnS nanoparticles without Manganese doping. XRD of the $\mathrm{Mn}^{2+}$ doped sample were carried out to know the average particle size.

90/10 (wt/wt) composition of PVA/ ZnS: Mn2+ is prepared for the experimental purpose. $5 \mathrm{wt} \%$ of PVA solution was prepared. Nanoparticles of $\left(\mathrm{Mn}^{2+}\right.$ approximately $10 \%$ concentrations) doped $\mathrm{ZnS}$ were prepared by chemical co-precipitation method as mentioned above and added to the PVA solution. The solution was kept for stirring for a day. The final solution was poured into a petridish and kept for drying. The film of the sample was obtained after a week of drying. Undoped ZnS in PVA matrix was also prepared.

\section{Results and discussion}

\subsection{XRD Analysis}

Fig. 1 shows the XRD pattern of the sample $\mathrm{ZnS}$ doped with $\mathrm{Mn}^{2+}$. The XRD pattern contain broad peak at $2 \theta=28.2^{\circ}$ indicating the formation of nanostructure. Using the Debye Scherrer formula

$$
\mathrm{D}=\frac{\mathrm{K} \lambda}{\beta \cos \theta}
$$

where $\theta$ is the Bragg's angle, $\lambda$ is the $\mathrm{Cu}-\mathrm{Ka}$ radiation wavelength, $\beta$ is the full width at half maxima, (here $K=0.89$ for spherical shape) the mean crystalline size (D) was calculated. The particles were assumed to be spherical. The calculated size is found to be 3.8 $\mathrm{nm}$. There are three diffraction peaks corresponding to diffraction angle $2 \theta$ of $28.28^{\circ}, 47.84^{\circ}$ and $55.98^{\circ}$ corresponding to (002), (110) and (112) lattice planes of hexagonal $\mathrm{ZnS}$ respectively. 
The small changes in diffraction angle are clearly a result of lattice contraction that is expected to occur because of higher surface to volume ratio [6].

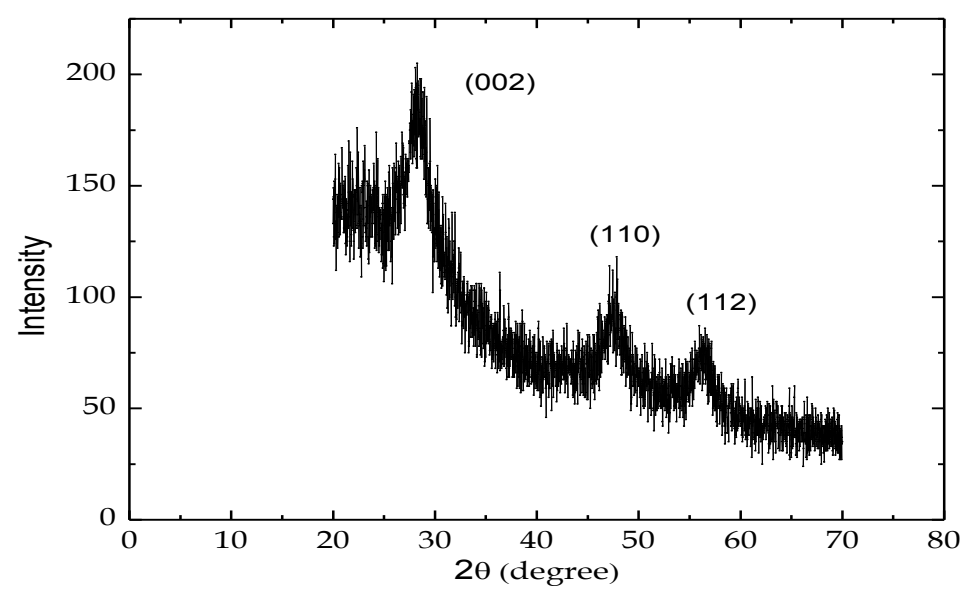

Fig. 1. XRD pattern of $\mathrm{ZnS}: \mathrm{Mn}^{2+}$ sample

\subsection{Optical studies}

The optical absorption of the samples is shown. The optical absorbances were recorded at the room temperature using UV-VIS spectrophotometer. The sample shows the peak at a wavelength $330 \mathrm{~nm}$ for pure ZnS, suggesting blue shift w.r.t. the bulk arising from quantum confinement effect in the nanoparticles. The absorption peak corresponding to the doped sample and the composites with PVA are listed in the Table 1.

The band gap of bulk $\mathrm{ZnS}$ is $3.68 \mathrm{eV}$ at $300 \mathrm{~K}$. The fundamental absorption, which corresponds to the transmission from valence band to the conduction band, is employed to determine the band gap of the material. The relation between absorption coefficient $(\alpha)$ and incident photon energy (hv) can be written as

$$
\alpha=\mathrm{A}\left(\mathrm{h} v-\mathrm{E}_{\mathrm{g}}\right)^{\mathrm{n}} / \mathrm{h} v
$$

where $\mathrm{A}$ is a constant and $\mathrm{E}_{\mathrm{g}}$ is the band gap of the material. Exponent ' $\mathrm{n}$ ' depends on the type of the transition; $\mathrm{n}$ may have 
values $1 / 2,2,3 / 2$ and 3 corresponding to the allowed direct, allowed indirect, forbidden direct and forbidden indirect transitions respectively. But in the nanocrystalline sample, there may be some deviation from the bulk like transition. From the above equation, it is clear that, plot of $(a h v)^{2}$ vs hv will indicate a divergence at an energy value $\mathrm{E}_{\mathrm{g}}$ where the transition takes place. Taking the values at discontinuities as the band gap, the nature of the transition (i.e., the $\mathrm{n}$ value) is determined. The exact value of the band gap is determined by extrapolating the straight line portion of $(a h v)^{2}$ vs hv graph to the $h v$ axis [7]. The values calculated using the above procedure is also tabulated in Table 1.

\begin{tabular}{|l|c|c|}
\hline \multicolumn{1}{|c|}{ Sample } & $\begin{array}{c}\text { Absorption Maxima } \\
(\mathrm{nm})\end{array}$ & $\begin{array}{c}\text { Energy Gap } \\
(\mathrm{eV})\end{array}$ \\
\hline $\mathrm{ZnS}$ & 330 & 3.2 \\
\hline $\mathrm{ZnS}: \mathrm{Mn}^{2+}$ & 250 & 4.3 \\
\hline PVA/ZnS & 320 & 3.2 \\
\hline PVA/ZnS: $\mathrm{Mn}^{2+}$ & 340 & 3.2 \\
\hline
\end{tabular}

Table 1: Energy gap and peak wavelength of the samples

\subsection{Photoluminescence studies}

Photoluminescence (PL) spectra for the samples were recorded using (JY Fluorolog-3-11) spectroflurometer. The PVA/ ZnS: $\mathrm{Mn}^{2+}$ nanocomposite samples are photoexcited at $320 \mathrm{~nm}$. Subsequent transfer of electron and hole into the electronic level of the Mn ion leads to the characteristic emission of $\mathrm{Mn}^{2+}$ in $\mathrm{ZnS}$. PL spectrum of composite contains an orange emission lying between 590-600 nm. The composite has a peak similar to pure $\mathrm{ZnS}: \mathrm{Mn} 2^{+}$nanoparticles $[8,9]$. 


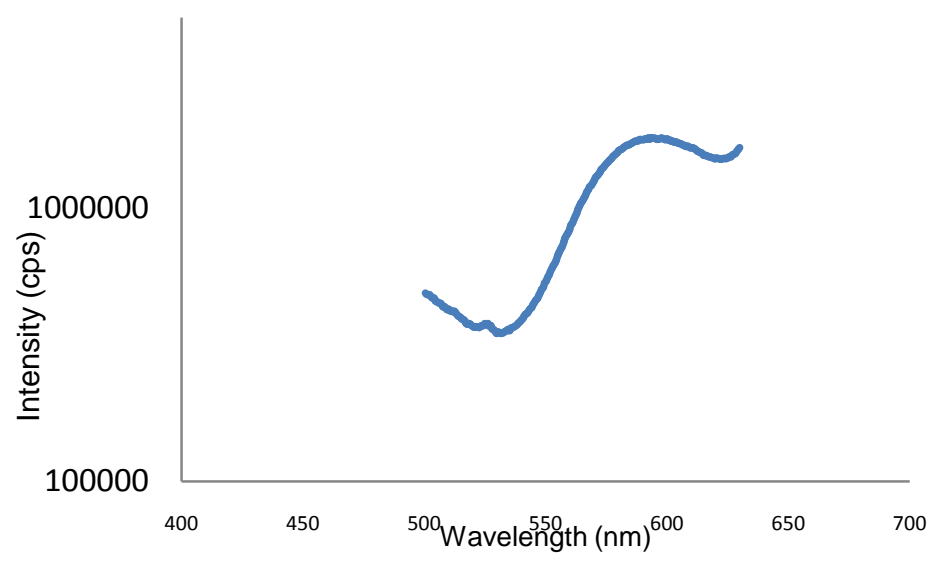

Fig. 2. Photoluminescence spectra of PVA/ ZnS: Mn2+ nanocomposite.

\section{Conclusion}

ZnS, ZnS:Mn2+, PVA/ZnS and PVA/ZnS:Mn²+ were synthesized by chemical co-precipitation method. The band gap of the samples was determined by UV-VIS spectra analyses. XRD analysis shows the $\mathrm{ZnS}$ sample prepared is in a hexagonal phase. The particle size is found to be $3.85 \mathrm{~nm}$. The composite shows an emission in the orange region as the $\mathrm{ZnS}: \mathrm{Mn}^{+}$samples.

\section{References}

[1] B. S. Rema Devi, R. Raveendran and A. V. Vaidyan, "Synthesis and characterization of $\mathrm{Mn}^{2+}$-doped $\mathrm{ZnS}$ nanoparticles," Pramana, vol. 679, p. 68, 2007.

[2] H. C. Warad, S. C. Ghosh, B. Hemtanon, C. Thanachayanont and J. Dutta, "Luminescent nanoparticles of Mn doped ZnS passivated with sodium hexametaphosphate," Scien. Technolog. Advan. Mater., vol. 6, p. 296, 2005. http://dx.doi.org/10.1016/j.stam.2005.03.006.

[3] J. C. Manifacier, M. D. Murcia, J. P. Fillard and E. Vicario, "Optical and electrical properties of $\mathrm{SnO} 2$ thin films in relation to their stoichiometric deviation and their crystalline structure," Thin Solid Films, vol. 41, p. 127, 1977.

[4] K. K. Nanda, S. N. Sarangi, and S. N. Sahu, "CdS Nanocrystalline films: Composition, surface, crystalline size, structural and optical 
absorption studies," Nanostruct. Mater. vol. 10, p. 1401, 1998. http:/ / dx.doi.org/10.1016/S0965-9773(99)00014-8.

[5] L. W. Wang and A. Zunger, "High-Energy Excitonic Transitions in CdSe Quantum Dots," J. Phy. Chem. B, vol. 102, p. 6449, 1998. http://dx.doi.org/10.1021/jp981018n.

[6] M. A. Khader and B. Thomas, "Dc conductivity of nanoparticles of CdS and ZnS," Nanostruct. Mater. vol. 10, p. 593, 1998. http:/ /dx.doi.org/10.1016/S0965-9773(98)00100-7.

[7] O. A. Korotchenkov, A. Canterero, A. P. Shpak, Y. A. Kunitskii, A. I. Senkevich, M. O. Borovoy and A. B. Nadtochii, "Doped ZnS:Mn nanoparticles obtained by sonochemical synthesis," Nanotechn., vol. 16, p. 2033, 2005. http://dx.doi.org/10.1088/0957-4484/16/10/008.

[8] S. K. Pandey, P. Verma and A. C. Pandey, Proc. ASID, vol. 259, p. 8, 2006.

[9] X. Fang, T. Zhai, U. K. Gautam, L. Li, L. Wu, Y. Bando and D. Goldberg, "ZnS nanostructures: From synthesis to applications," Prog. Mater. Scien., vol. 56, p. 175, 2011. http://dx.doi.org/ 10.1016/j.pmatsci.2010.10.001. 